\title{
Fertilization with beneficial microorganisms decreases tomato defenses against insect pests
}

\author{
Lea Megali • Gaétan Glauser • Sergio Rasmann
}

Accepted: 1 October 2013 / Published online: 25 October 2013

(C) INRA and Springer-Verlag France 2013

\begin{abstract}
The adverse effects of chemical fertilizers on agricultural fields and the environment are compelling society to move toward more sustainable farming techniques. "Effective microorganisms" is a beneficial microbial mixture that has been developed to improve soil quality and crop yield while simultaneously dramatically reducing organic chemical application. Additional indirect benefits of beneficial microorganisms application may include increased plant resistance to herbivore attack, though this has never been tested till now. Tomato plants were grown in controlled greenhouse conditions in a full-factorial design with beneficial microorganisms inoculation and commercial chemical fertilizer application as main factors. We measured plant yield and growth parameters, as well as resistance against the generalist pest Spodoptera littoralis moth larval attack. Additionally, we measured plant defensive chemistry to underpin resistance mechanisms. Overall, we found that, comparable to chemical fertilizer, beneficial microorganisms increased plant growth fruit production by 35 and $61 \%$, respectively. Contrary to expectations, plants inoculated with beneficial microorganisms sustained $25 \%$ higher insect survival and larvae were in average $41 \%$ heavier than on unfertilized plants. We explain these results by showing that beneficial microorganisminoculated plants were impaired in the induction of the toxic
\end{abstract}

L. Megali · S. Rasmann

Department of Ecology and Evolution, University of Lausanne

(UNIL), Sorge, Le Biophore, 1015 Lausanne, Switzerland

G. Glauser

Chemical Analytical Service of the Swiss Plant Science Web,

University of Neuchatel, 2000 Neuchatel, Switzerland

Present Address:

S. Rasmann $(\bowtie)$

Department of Ecology and Evolutionary Biology, University of

California, 92697 Irvine, CA, USA

e-mail: sergio.rasmann@unil.ch glycoalkaloid molecule tomatine and the defense-related phytohormone jasmonic acid after herbivore attack. For the first time, we therefore show that biofertilizer application might endure unintended, pest-mediated negative effects, and we thus suggest that biofertilizer companies should incorporate protection attributes in their studies prior to commercialization.

Keywords Chemical fertilizer · Induced resistance $\cdot$ Insect herbivore $\cdot$ Phytohormone $\cdot$ Plant defense $\cdot$ Plant-microbe interaction $\cdot$ Sustainable agriculture

\section{Introduction}

Agriculture is the primary source of human nutrition, and rapid and ever-increasing human population growth has led to the extreme intensification of agricultural systems. To address the increasing yield demand with decrease in available agricultural land, farmers practicing conventional agriculture have been compelled to apply continually higher doses of harmful chemical fertilizers and pesticides (Foley et al. 2011). Soil pollution, particularly, that is caused by the excessive application of fertilizers and pesticides, has been identified as a priority that requires resolution within the next decade (Wardle et al. 2004). In other words, there is much concern to further preserve environmental integrity and public health through the use of less intensive and more sustainable agricultural practices by reducing the inputs of chemical fertilizers, pesticides, and energy demand in general (Gomiero et al. 2011).

Diverse groups of soil-borne microbes, such as root endophytic fungi, mycorrhizal fungi, plant growth-promoting rhizobacteria, and rhizobia, exert positive effects on plant growth and survival through direct and plant-mediated mechanisms (Compant et al. 2005; Van der Heijden et al. 2008; 
Pineda et al. 2010). Although mycorrhizal fungi and arbuscules have long been acknowledged to increase plant growth (Van der Heijden et al. 2008), more recent evidence highlights the roles of plant growth-promoting rhizobacteria, root endophytic fungi, and plant growth-promoting fungi for increasing plant performance (Compant et al. 2005). Therefore, integrating beneficial microorganisms as biofertilizers is attracting growing interest in sustainable agriculture (Javaid 2010). Numerous studies have additionally demonstrated that the association with soil-containing microbes can increase the resistance of plants against below-ground attack, such as that caused by soilborn fungi, bacterial pathogens, and nematodes, or aboveground herbivore attack and shoot pathogens (reviewed in Azcón-Aguilar and Barea 1996; Pozo and Azcon-Aguilar 2007; Pineda et al. 2010).

The aims of this study were therefore to test whether a commercial organic microbial soil inoculum (effective microorganisms; EM; Higa and Parr 1994) are able to firstly, increase crop yields as previously shown for other systems (e.g., Javaid 2006, 2011; Javaid and Bajwa 2011a, b), and secondly, simultaneously improve plant resistance to herbivore attack (Fig. 1a, b). We tested these hypotheses using a series of controlled-environment experiments with tomato plants, and through the full factorial manipulation of soil inoculation with the beneficial microorganisms or with conventional chemical fertilizer. We expected that the plants inoculated with the beneficial microorganisms would perform as good as when inoculated with chemical fertilizers. Second, we expected that beneficial microorganism-inoculated plants would show increased resistance against herbivores in comparison to the control or chemically inoculated plants.

\section{Material and methods}

\subsection{Effective microorganisms}

The beneficial microorganisms mixture, developed in the early 1990s by Professor Teruo Higa from the University of the Ryukyus, Okinawa, Japan (Higa and Parr 1994), is mainly composed of lactic acid bacteria (viz., Lactobacillus plantarum, Lactobacillus casei, and Streptococcus lactis), yeasts (Saccharomyces spp.), phototrophic bacteria (viz., Rhodopseudomonas plastris and Rhodobacter sphacrodes), and actinomycetes (Strptomyces spp.; Cóndor Golec et al. 2007; Higa 2000; Hussain et al. 2002). The mixture has been shown to improve crop health and yield by increasing the photosynthetic rate, and by accelerating soil decomposition of organic matter and the release of nutrients for plant uptake (Hussain et al. 1999). The microbial mixture is commercially available in Switzerland as a concentrated liquid suspension (EM-1), which is the base substrate for the following different EM preparations based on anaerobic fermentation: (a) with

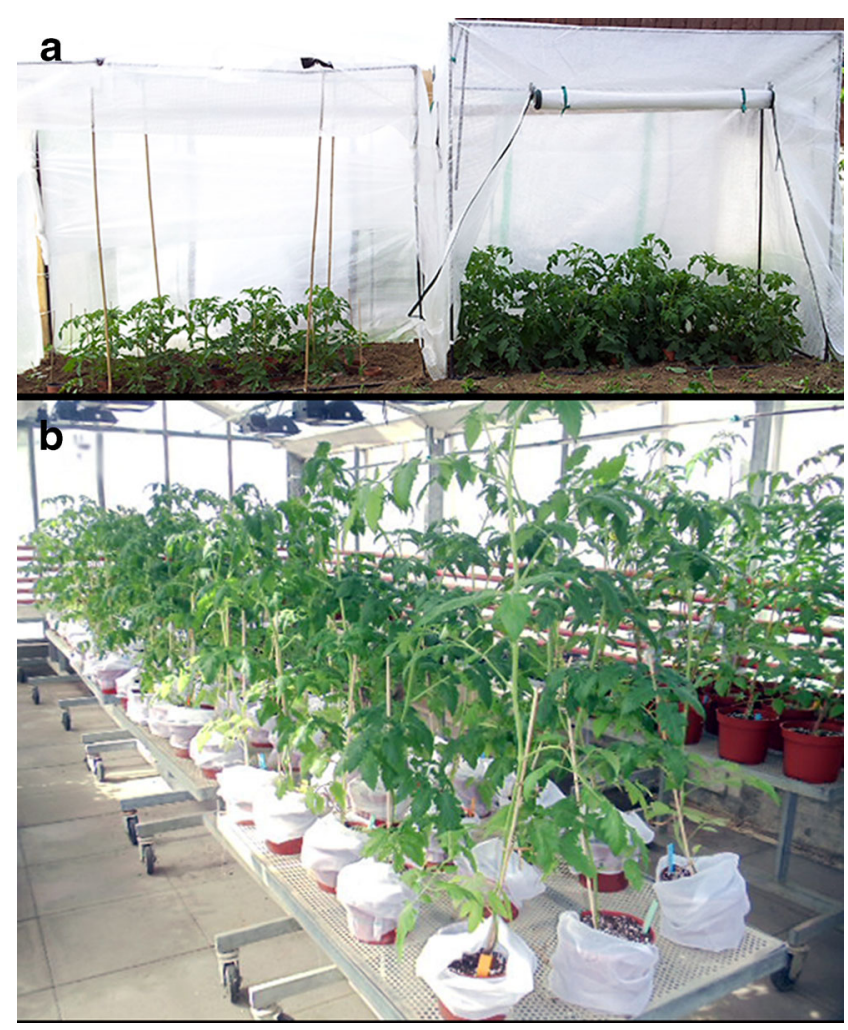

Fig. 1 Tomato plants experimental design. a The preliminary study of tomato plants placed into two portable field greenhouses placed side-byside that were either left unfertilized (no beneficial microorganisms or no NPK addition, left ) or on the right side, the soil was inoculated with $3 \mathrm{~kg}$ of beneficial microorgnaims (EM-Bokashi, EM Schweitz AG, Switzerland) per square meter. Beneficial microorganisms application increased the plant biomass by $47 \%$ compared to the unfertilized plants. b The greenhouse setting used to perform all the described experiments in the text. The four treatments (control unfertilized plants, plants fertilized with beneficial microorganisms, plants fertilized with NPK, and plants fertilized with both beneficial microorganisms and NPK) were randomly distributed on the benches within the greenhouse. For experiments shown in Figs. 2c, d and 3, half of the plants were damaged by the noctuid butterfly Spodoptera littoralis. Insects were prevented escaping with fine-meshed nylon bags around the plants (shown folded down here)

sugarcane molasses and water (EMA); (b) with sugarcane molasses, water, ethanol, and vinegar (EM5); and (c) with sugarcane molasses and a fermentable organic substrate (Bokashi). EMA and EM5 are used as spraying agents and a combination of Bokashi (www.EM-schweiz.ch/; date of release 26 November 2012). All our experiments were performed using Bokashi mixed with potting soil, and EM-1 as liquid soil inoculation (see below).

\subsection{Plant growth and performance experiments}

A preliminary study using cherry tomato plants (Solanum lycopersicum) that were grown in two different portable field greenhouses with and without EM-1 clearly showed that beneficial microorganisms inoculation increases plant growth and yield (Fig. 1a). We therefore next tested the same variety 
of plants (cherry tomatoes from Landi AG, Switzerland) in a controlled greenhouse setting. Seeds were germinated in autoclaved medium-low $\mathrm{P}$ potting soil (Orbo-2, Schweizer AG, Lausanne; Switzerland) with perlite (3:1). After 12 days, same-sized plantlets were transplanted into $15-\mathrm{cm}$ diameter plastic pots containing the same soil as for the germination and placed in a greenhouse at $25 / 18{ }^{\circ} \mathrm{C}, 60 \%$ relative humidity, and a photoperiod consisting of $14 \mathrm{~h}$ of daylight. The plants were next separated into four treatments: (1) control, untreated plants; (2) beneficial microorganism-treated plants; (3) synthetic fertilizer-treated plants (NPK); and (4) both beneficial microorganisms and NPK-treated plants. According to the vendor recommendation, the beneficial microorganisms treatment consisted of $20 \mathrm{~g}$ of the solid Bokashi commercial preparation (EM Schweitz AG, Bern, Switzerland) placed at the bottom of the pots prior to transplanting the germinated seeds. In addition, $50 \mathrm{ml}$ of Bokashi liquid $(1: 1,000)$ was applied once a week. Chemical fertilizer (NPK) (50 ml of a $1 \%$ preparation, Landi, Switzerland) was applied once a week. An additional $50 \mathrm{ml}$ of tap water was added to each pot during the treatment application to mimic the double treatment (Bokashi plus NPK).

The treatments were applied using a complete randomized design with 15 replicates in each treatment. The performance of the tomato plants was analyzed with four different measures: (a) the levels of chlorophyll measured three times per leaf and for three leaves per plant using a SPAD-502Plus chlorophyll meter (Konica Minolta (China) Investment Ltd) at 50 days after planting; (b) plant dry biomass (after 5 days at $60{ }^{\circ} \mathrm{C}$ ); (c) number of flowers (measured twice at 56 and 105 days after transplanting); and (d) number of fruits (measured twice at 85 and 105 days after seedling).

All the response variables related to growth and yield were analyzed using a two-way analysis of variance (ANOVA), with the beneficial microorganisms application (two levels), NPK application (two levels), and their interaction as the main effects. Student's $t$ tests were used to assess in-between treatment differences $(p<0.05)$.

\subsection{Plant resistance and defense experiments}

To analyze the effect of beneficial microorganisms on plant defenses against herbivore attack, the same conditions and design as described above were used, except that 20 replicates were applied instead of 15 , providing five more replicates for the chemical analysis of undamaged control plants. At 40 days after transplanting, 20 Spodoptera littoralis larvae were added to each of 15 plants per treatment. The Egyptian cotton leafworm $S$. littoralis is a highly polyphagous nocturnal moth (Lepidoptera: Noctuidae) originating from Northern Africa and the Mediterranean Basin and is a major pest of crop plants, including cotton, corn, and tomato. The entire plant and pot were then covered with a fine-meshed nylon net to prevent the larvae from escaping. The larvae were allowed to feed for 1 week after which they were harvested and flash frozen in liquid nitrogen, and the larval mortality per plant was recorded. The larvae were then dried at room temperature and weighted as a measure of growth rate. The nylon mesh, but not herbivores, was applied to the control plants.

Subsequently, the leaf tissues of all plants were collected to measure the beneficial microorganism effect on the defensive chemical secondary metabolites and phytohormones of tomato. Tomato plants are known to produce several toxic metabolites to deter herbivore attack, including the highly toxic glycoalkaloid tomatine (Duffey and Stout 1996). Therefore, we quantified tomatine in the leaves using ultra high performance liquid chromatography (UHPLC)-quadrupole-time-of-flight mass spectrometry (QTOFMS). About $20 \mathrm{mg}$ of fresh leaves samples were harvested, flash frozen, and ground with $1 \mathrm{ml}$ of solvent ( $80 \% \mathrm{MeOH}, 20 \% \mathrm{H}_{2} \mathrm{O}$, and $0.5 \%$ formic acid). After mixing and centrifugation at14,000 rpm for $3 \mathrm{~min}$, the supernatant was removed and diluted 50-fold with the extraction solvent prior to the analysis. The UHPLC separation was performed in gradient mode under the following conditions: solvent A, water $+0.05 \%$ formic acid; solvent $\mathrm{B}$, acetonitrile + $0.05 \%$ formic acid; $5-40 \% \mathrm{~B}$ in $3.5 \mathrm{~min}, 40-100 \% \mathrm{~B}$ in $1.0 \mathrm{~min}$, holding at $100 \% \mathrm{~B}$ for $1.0 \mathrm{~min}$, and re-equilibration at $5 \% \mathrm{~B}$ for $1.0 \mathrm{~min}$. The column was an Acquity BEH C18 (50× $2.1 \mathrm{~mm}$ i.d., $1.7 \mu \mathrm{m}$ particle size, Waters Corp., Milford, MA, USA). The flow rate was set to $400 \mu \mathrm{L} / \mathrm{min}$, and the injection volume was $2.0 \mu \mathrm{L}$. The mass spectrometer was operated in electrospray positive mode. The final concentration of tomatine was expressed in $\mu \mathrm{g} / \mathrm{g} \times \mathrm{FW}$ based on external calibration.

Lastly, we measured phytohormone accumulation in the healthy and damaged plants according to Glauser et al. (2014); specifically, we measured the jasmonic acid and salicylic acid contents of the plants. Both hormones accumulate and orchestrate defenses against biotic attack. Jasmonic acid mainly mediates herbivore attack (Howe 2004), whereas salicylic acid mainly mediates pathogen attack (Ton et al. 2002); additionally, the two hormones are proposed to antagonize each other's pathway (Thaler et al. 2012). The extraction of phytohormones was performed by grinding $200 \mathrm{mg}$ of fresh leaves to a powder and mixing with $990 \mu$ of extraction solvent (ethylacetate/formic acid, 99.5:0.5) and $10 \mu \mathrm{l}$ of internal standards (ISs; containing isotopically labeled hormones at a concentration of $100 \mathrm{ng} / \mathrm{mL}$ for $\mathrm{d}_{5}-\mathrm{JA}, \mathrm{d}_{6}-\mathrm{SA}$ ) in a mixer mill at $30 \mathrm{~Hz}$. After centrifugation and evaporation of the supernatant, the residue was resuspended in $100 \mu \mathrm{L} 70 \% \mathrm{MeOH}$. Five $\mu$ l of the solution was injected for UHPLC-MS/MS analysis following the same conditions as in Glauser et al. (2014). The final concentration of the two phytohormones, salicylic acid (SA), and jasmonic acid (JA) (expressed in ng/ $\mathrm{g} \times \mathrm{FW}$ ), was extrapolated for each sample using calibration curves in which the ISs were present at the same concentrations as in the plant samples. 
The larval performance parameters (biomass gained and survival) were analyzed with a two-way ANOVA, with the beneficial microorganisms application (two levels), NPK application (two levels), and their interaction as the main effects. The plant compounds (tomatine and phytohormones) were analyzed with a three-way ANOVA, with the two same soil inoculation factors as above plus the herbivore treatment (two levels) and all the interaction combinations as the main effects. Biomass and chemical compounds data were log-transformed and survival was square root-transformed prior to analyses to meet homoscedasticity assumptions. Student's $t$ tests were used to assess in-between treatment differences $(p<0.05)$.

\section{Results and discussion}

\subsection{Plant growth and performance}

The fertilization treatments significantly increased the growth and yield of the tomato plants. The plants grown with beneficial microorganisms or NPK exhibited 16 and $35 \%$, respectively, more biomass than the control plants (Fig. 2a). The leaves from the beneficial microorganism-treated plants contained $11 \%$ more chlorophyll than the leaves from the control plants and $5 \%$ more chlorophyll than the NPK-treated plants (Fig. 2b, Table 1). Additionally, we found that the beneficial microorganism treatment significantly increased the timing of flowering and fruit production (Table 1). After 56 days from seedling, the plants treated with beneficial microorganisms contained an average of 69 and $50 \%$ more flowers than the control and NPK plants, respectively. Similarly, after 85 days from seedling, the plants treated with beneficial microorganisms contained $61 \%$ more fruits than the control plants and $38 \%$ more fruits than the plants inoculated with NPK.

These results are in agreement with several previous tests of beneficial microorganisms efficacy on crop plants. According to our own literature survey, across 36 independent studies and 26 plant species tested, beneficial microorganisms (EM) soil application increases plant yield by $30 \%$ (Megali and Rasmann, unpublished). However, such overall positive results remain largely debated, as contradictory or no effects of the application of beneficial microorganisms preparations on crop yields and plant development have also been reported (e.g., Mayer et al. 2010; Iwaishi 2001; Xu et al. 2000; Javaid and Shah 2010; van Vliet et al. 2006; Okorski et al. 2010). As an example, and most likely one of the most thorough studies to date, Mayer et al. (2010) showed that beneficial microorganisms application alone did not significantly increase crop yield, even though such effects ranged from $0.4 \%$ increase in
Fig. 2 Organic and chemical fertilizer effect on a plant biomass and $\mathbf{b}$ chlorophyll levels for the plants that were either left unfertilized (control), fertilized with commercial chemical fertilizer (NPK), inoculated with beneficial microorganism (EM-1 from EM Schweitz AG) (EM), or inoculated with both chemical and organic fertilizer (NPK+ EM). Bottom panels show the same soil treatment effects on $\mathbf{c}$ Spodoptera littoralis

(Lepipdotera; Noctuidae) survival and $\mathbf{d} S$. littoralis biomass gained (dry weight) after 1 week of feeding. Bars are average $\pm 1 \mathrm{SE}$, letters above the bars indicate significant differences among the soil treatments $(P<0.05$ Student's $t$ test)
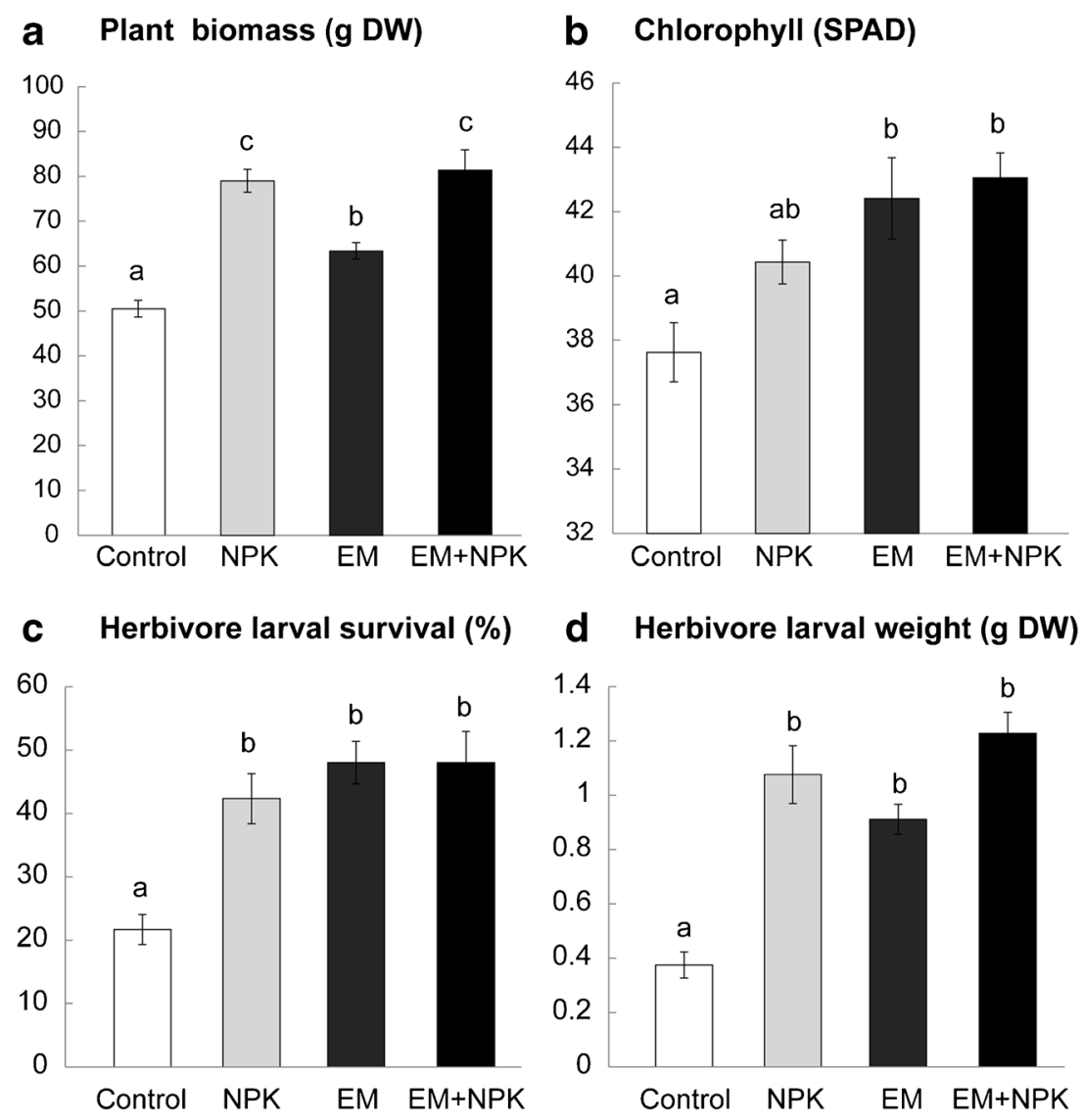
Table 1 Two-way ANOVA table for the effect of beneficial microorganisms and chemical fertilizer (NPK) soil application on plant growth and performance traits. The bold font indicates a significant effect $(P<0.05)$. $\mathrm{Df}_{\text {num }}=1$

\begin{tabular}{|c|c|c|c|c|}
\hline Response & Factor & $D f_{\text {den }}$ & $F_{\text {value }}$ & $P$ \\
\hline \multirow[t]{3}{*}{ Chlorophyll } & Beneficial microorganisms & 54 & 14.502 & $<0.001$ \\
\hline & NPK & & 3.032 & 0.087 \\
\hline & Beneficial microorganisms $\times \mathrm{NPK}$ & & 0.245 & 0.662 \\
\hline \multirow[t]{3}{*}{ Plant biomass } & Beneficial microorganisms & 45 & 8.848 & 0.010 \\
\hline & NPK & & 65.925 & $<0.0001$ \\
\hline & Beneficial microorganisms $\times \mathrm{NPK}$ & & 3.350 & 0.073 \\
\hline \multirow[t]{3}{*}{ Number of flowers (56 days after seedling) } & Beneficial microorganisms & 54 & 13.784 & $<0.001$ \\
\hline & NPK & & 4.416 & 0.040 \\
\hline & Beneficial microorganisms $\times \mathrm{NPK}$ & & 3.687 & 0.0635 \\
\hline \multirow[t]{3}{*}{ Number of flowers (105 days after seedling) } & Beneficial microorganisms & 47 & 6.549 & 0.013 \\
\hline & NPK & & 36.234 & $<0.0001$ \\
\hline & Beneficial microorganisms $\times$ NPK & & 1.958 & 0.168 \\
\hline \multirow[t]{3}{*}{ Number of fruits ( 85 days after seedling) } & Beneficial microorganisms & 47 & 6.413 & 0.014 \\
\hline & NPK & & 1.787 & 0.187 \\
\hline & Beneficial microorganisms $\times$ NPK & & 25.325 & $<0.001$ \\
\hline \multirow[t]{3}{*}{ Number of fruits (105 days after seedling) } & Beneficial microorganisms & 56 & 0.354 & 0.554 \\
\hline & NPK & & 0.083 & 0.774 \\
\hline & Beneficial microorganisms $\times \mathrm{NPK}$ & & 3.762 & 0.057 \\
\hline
\end{tabular}

yield for lucerne plants to $21 \%$ for potato plants. Overall, the strongest effect was observed when the microbial solution was applied in conjunction with the Bokashi organic substrate, therefore, the authors argue that the addition of mineral and carbon sources via Bokashi application is sufficient to increase crop yield, whereas the microorganisms do not have any measurable impact on the plants (Mayer et al. 2010). We found a positive effect of beneficial microorganisms application on the photosynthetic potential, as did others (Xu 2001; Okorski et al. 2010). Similarly, these authors also indicated that the significant positive effect of beneficial microorganisms on plant chlorophyll levels could be attributed to the Bokashi carrier substrate and not beneficial microorganisms microbial mixture alone. In our study, we could not separate the direct effect of organic substrate from the microbial application alone; therefore, we cannot exclude a large effect of Bokashi organic amendment application at the bottom of the pots for increasing the crop yield.

Lastly, the plants treated with beneficial microorganisms produced more flowers and at an earlier time than the NPK- and NPK plus beneficial microorganism-treated or control plants, and this led the beneficial microorganismtreated plants to produce fruits earlier than the plants in the other treatments. This conclusion is supported by an experiment with paddy rice in which the flowering and fruiting of plants treated with beneficial microorganisms were 710 days earlier than rice grown using conventional methods (Lin 1991).
The exact mechanisms responsible for the increased benefits due to beneficial microorganisms are largely obscure but include increased soil mineralization (Daly and Stewart 1999) and modification of the bacterial and fungal soil community structure (Mayer et al. 2010; Dilly and Blume 1996). Schenck zu Schweinsberg-Mickan and Müller (2009) attribute the positive effect of increased soil respiration and the net $\mathrm{N}$ immobilization to molasses, which is the carrier substrate of EMA and not beneficial microorganisms alone. Nevertheless, independent of the exact mechanisms, we can posit that, when applied to crop fields, beneficial microorganisms, and organic substrate together have a strong potential to increase plant performance and yield and have a similar effect at chemical fertilizer application. Future research should further aim at isolating the different microbial groups, and measuring the individual as well as their combined effects.

\subsection{Plant resistance and defense}

S. littoralis Larval survival was the lowest on the control plants, with only $20 \%$ of the larvae surviving compared to $45 \%$ survival for the average of the other soil inoculation treatments (Fig. 2c). Similarly, the larval dry biomass was higher for larvae feeding on the beneficial microorganisms, NPK, or beneficial microorganisms plus NPK-fertilized plants than the larvae that grew on the control plants (Fig. 2d). On average, the larvae that fed on the beneficial microorganisms, NPK, or both beneficial microorganisms and NPK plants were 


\section{a Tomatine concentration in leaves $(\mu \mathrm{g} / \mathrm{g})$}

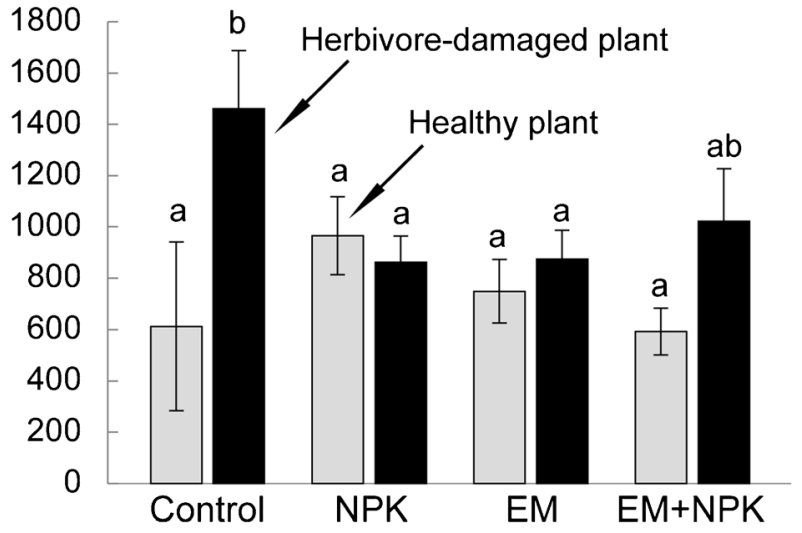

b Jasmonic acid concentration in leaves (ng/g)

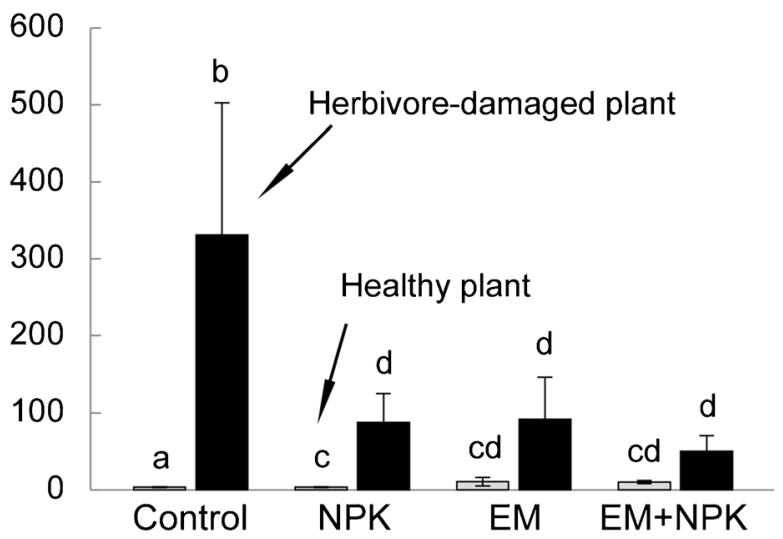

\section{Salicylic acid concentration in leaves (ng/g)}

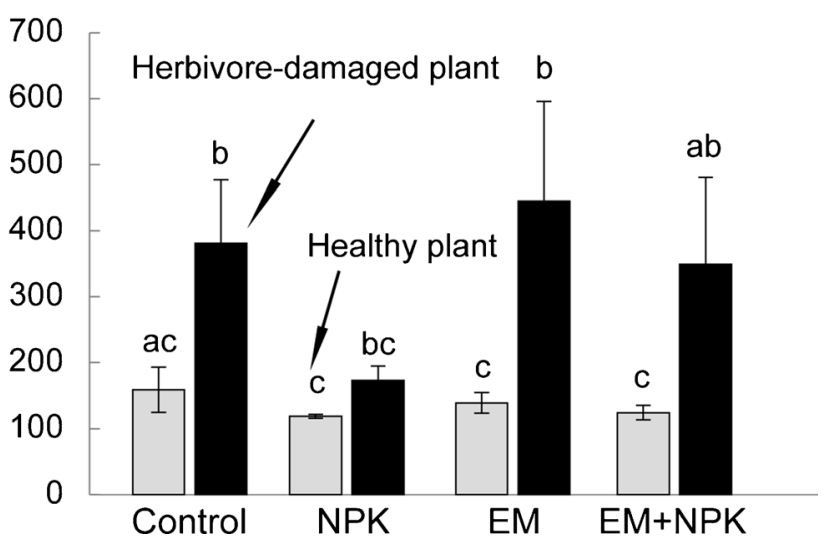

Fig. 3 Organic and chemical fertilizer effect on plant defense deployment. Shown is the average $\pm 1 \mathrm{SE}$ of a tomatine content, $\mathbf{b}$ jasmonic acid, and $\mathbf{c}$ salicylic acid content in plants that were either left unfertilized (control), fertilized with commercial chemical fertilizer (NPK), inoculated with beneficial microorganism (EM Bokashi and EM-1 from EM Schweitz AG) (EM), or inoculated with both chemical and organic fertilizer (NPK+EM). Additionally, half of the plants were infested with 10 Spodoptera littoralis caterpillars for 4 days (black bars) or left undamaged (gray bars). The letters above the bars indicate significant difference among the soil treatments $(P<0.05$ Student's $t$ test $)$
Table 2 Three-way ANOVA table for the effect of beneficial microorganisms and chemical fertilizer (NPK) soil application and herbivore induction on plant defense chemistry (tomatine) and phytohormones (jasmonic acid and salicylic acid). The bold font indicates significant effect $(P<0.05)$. $D f=1.27$

\begin{tabular}{llrc}
\hline Response & Factor & $F_{\text {value }}$ & $P$ \\
\hline Tomatine & Beneficial microorganisms & 1.135 & 0.296 \\
& NPK & 0.002 & 0.967 \\
& Beneficial microorganisms $\times$ NPK & 0.115 & 0.737 \\
& Herbivore $(\mathrm{H})$ & 8.805 & $\mathbf{0 . 0 0 6}$ \\
& Beneficial microorganisms $\times \mathrm{H}$ & 0.183 & 0.672 \\
& NPK $\times \mathrm{H}$ & 2.038 & 0.165 \\
Beneficial microorganisms $\times \mathrm{NPK} \times \mathrm{H}$ & 7.322 & $\mathbf{0 . 0 1 2}$ \\
Jasmonic acid & beneficial microorganisms & 0.006 & 0.9402 \\
& NPK & 1.410 & 0.2458 \\
& Beneficial microorganisms $\times \mathrm{NPK}$ & 1.731 & 0.200 \\
& Herbivore $(\mathrm{H})$ & 99.233 & $<0.0001$ \\
& Beneficial microorganisms $\times \mathrm{H}$ & 10.796 & 0.003 \\
NPK $\times \mathrm{H}$ & 3.503 & 0.073 \\
Beneficial microorganisms $\times \mathrm{NPK} \times \mathrm{H}$ & 0.702 & 0.410 \\
Salicylic acid & Beneficial microorganisms & 0.847 & 0.366 \\
& NPK & 4.916 & $\mathbf{0 . 0 3 5}$ \\
Beneficial microorganisms $\times \mathrm{NPK}$ & 0.889 & 0.354 \\
Herbivore $(\mathrm{H})$ & 26.721 & $<\mathbf{0 . 0 0 0 1}$ \\
Beneficial microorganisms $\times \mathrm{H}$ & 1.314 & 0.262 \\
NPK $\times \mathrm{H}$ & 1.148 & 0.294 \\
Beneficial microorganisms $\times \mathrm{NPK} \times \mathrm{H}$ & 0.298 & 0.590 \\
\hline & & &
\end{tabular}

41,55 , and $66 \%$ heavier, respectively, than the larvae that fed on the control plants (Fig. 2d).

Herbivores increased tomatine concentration in the leaves but only for the plants not receiving fertilizer (Fig. 3a, Table 2). Both the chemical fertilizer and beneficial microorganisms application inhibited the plant responses to herbivore attack and did not increase the leaf tomatine content, which was reflected by a poor induction of JA in all the soil application treatments, except for the control, non-inoculated plants (Fig. 3b, Table 2). In contrast, $S$. littoralis feeding significantly induced salicylic acid in the plants of all treatments, particularly with beneficial microorganisms and with both beneficial microorganisms and NPK inoculation (Fig. 3c, Table 2).

In contrast to the predictions of this study, none of the results suggested that beneficial microorganisms soil application could increase plant-resistant traits against herbivore attacks. Firstly, the $S$. littoralis larvae grew better on the plants with beneficial microorganisms and NPK or the plants treated with beneficial microorganisms plus NPK than the control plants. This result was consistent with the lower mortality of larvae found for the beneficial microorganisms or NPK plants. 
As described above, the bacteria present in the beneficial microorganisms preparation increase organic matter mineralization, leading to a better soil quality and, indirectly, a more palatable plant (Daly and Stewart 1999; Higa and Parr 1994; Lin 1991). Therefore, because a resource-rich plant is more attractive and palatable to herbivores than a plant lacking in nutrients, this could explain the higher insect performance on the fertilized plants. Interestingly, we did not detect variation in carbon to nitrogen ratio across treatments (results not shown) suggesting that fertilizer-mediated variation in resistance is mainly driven by toxic secondary metabolite production. Indeed, we could show that fertilized plants under herbivore attack cannot increase their levels of defenses (tomatine). Our findings are in agreement with classic defense hypotheses, which suggest that slow-growing plants on nutrient poor soils should invest in higher levels of defense and vice versa (Coley et al. 1985). We could also mechanistically show that such trade-offs between growth and defense are mediated by the phytohormonal inhibition of defense induction. In particular, jasmonic acid is associated with the orchestration of defenses against chewing herbivores (Thaler et al. 2012), such as $S$. littoralis caterpillars in our case, and its induction is inhibited when soils are fertilized with either chemical or organic soil additions. Interestingly, we observed that, although jasmonic acid was inhibited, the activity of the hormone salicylic acid remained intact in soil-rich environments. Antagonism and negative effects between these two phytohormones have been classically postulated (Thaler et al. 2012) and could explain our results.

Besides directly defending themselves by producing toxic compounds such as tomatine in tomatoes, plants can indirectly defend themselves by attracting natural enemies of herbivores via the production of volatile signals (Dicke and Baldwin 2010). Corn plants that were inoculated with the same doses of beneficial microorganisms used in the present study were recently observed to exhibit a similar inhibition of volatile organic compound production (Megali and Rasmann, unpublished), suggesting that both direct and indirect defenses are inhibited when soils are fertilized.

Therefore, we cannot exclude that other nonmeasured toxic molecules were less abundant in fertilized plants, justifying further research on the topic. Lastly, plants can simply resist herbivores by increasing their ability to regrow after attack. Indeed, by improving nutrient and water uptake, beneficial microbes can facilitate the regrowth of chewed tissues and thus promote plant tolerance in the presence of chewing insects (Bennett et al. 2006). To verify this hypothesis, it would be interesting to apply herbivores to plants treated with beneficial microorganisms and plants without beneficial microorganisms to compare the efficiency of the regrowth of damaged tissues and the final plant biomass and yield.

\section{Conclusion}

Across several experiments, we showed that soil microbial inoculation increased plant performance and accelerated fruit production in a manner that was as good as, or even better, than with the application of chemical fertilizers. However, both the bio- and the chemical fertilizer soil applications induced susceptibility by making the plants more palatable to aboveground insect herbivore. These results were due to the fertilizers inhibiting the ability of the plants to increase their chemical defenses when under attack. However, how soil microbes impact higher trophic levels through the modification of the plant physiology has strong specificity (Pineda et al. 2010). For instance, the chrysomelid beetle Diabrotica speciosa fed less on beneficial microorganism-treated plants compared to control-untreated plants (Ursi Ventura et al. 2006). This suggests a specificity of the soil quality dependence for the maintenance or breakdown of the trade-off between growth and defense, and demands community-wide measures of the microbes' effect on plant traits. We thus suggest that biofertilizer companies should incorporate their product-mediated protection attributes against pests in their studies prior to commercialization.

Acknowledgments This project was supported by Swiss National Science Foundation Ambizione grant PZ00P3 131956/1 to SR. We are grateful to Ueli Rothenbuhler for providing the microbial substrate, to Julia Bilat and Dr. Armelle Vallat for laboratory analyses, and to Eros Gentilini for stimulating the ideas that prompted this study.

\section{References}

Azcón-Aguilar C, Barea JM (1996) Arbuscular mycorrhizas and biological control of soil-borne plant pathogens - an overview of the mechanisms involved. Mycorrhiza 6(6):457-464

Bennett AE, Alers-Garcia J, Bever JD (2006) Three-way interactions among mutualistic mycorrhizal fungi, plants, and plant enemies: hypotheses and synthesis. Am Nat 167(2):141-152

Coley PD, Bryant JP, Chapin FS (1985) Resource availability and plant antiherbivore defense. Science 230(4728):895-899

Compant S, Duffy B, Nowak J, Clément C, Barka EA (2005) Use of plant growth-promoting bacteria for biocontrol of plant diseases: principles, mechanisms of action, and future prospects. Appl Environ Microbiol 71(9):4951-4959. doi:10.1128/aem.71.9.4951-4959.2005

Cóndor Golec AF, Lokare C, González Pérez P (2007) Effective microorganisms: myth or reality? Rev Peru Biol 14(2):315-319

Daly MJ, Stewart DPC (1999) Influence of "effective microorganisms" (EM) on vegetable production and carbon mineralization - a preliminary investigation. J Sustainable Agric 14(2-3):15-25

Dicke M, Baldwin IT (2010) The evolutionary context for herbivoreinduced plant volatiles: beyond the 'cry for help'. Trends Plant Sci 15(3):167-175. doi:10.1016/j.tplants.2009.12.002

Dilly O, Blume HP (1996) Indicators to assess sustainable land use with reference to soil microbiology. In: Blume HP, Eger H, Fleischhauer E, Hebel A, Reij C, Steiner KG (eds) 9th Conference of the 
International-Soil-Conservation-Organisation. Catena, Bonn, Germany, pp 29-36

Duffey SS, Stout MJ (1996) Antinutritive and toxic components of plant defense against insects. Arch Insect Biochem Physiol 32(1):3-37

Foley JA, Ramankutty N, Brauman KA, Cassidy ES, Gerber JS, Johnston M, Mueller ND, O’Connell C, Ray DK, West PC, Balzer C, Bennett EM, Carpenter SR, Hill J, Monfreda C, Polasky S, Rockstrom J, Sheehan J, Siebert S, Tilman D, Zaks DPM (2011) Solutions for a cultivated planet. Nature 478(7369):337-342. doi:10.1038/ nature 10452

Glauser G, Vallat A, Balmer D (2014) Hormone profiling. In: SanchezSerrano JJ, Salinas J (eds) Arabidopsis protocols, methods in molecular biology, vol 1062. Springer, Netherlands, pp 597-608. doi: 10.1007/978-1-62703-580-4_31

Gomiero T, Pimentel D, Paoletti MG (2011) Is there a need for a more sustainable agriculture? Crit Rev Plant Sci 30(1-2):6-23. doi:10. 1080/07352689.2011.553515

Higa T (2000) What is EM technology? EM World Journal 1:1-6

Higa T, Parr J (1994) Beneficial and effective microorganisms for a sustainable agriculture and environment. International Nature Farming Research Center, Atami, Japan

Howe GA (2004) Jasmonates as signals in the wound response. J Plant Growth Regul 23(3):223-237. doi:10.1007/s00344-004-0030-6

Hussain T, Javaid T, Parr JF, Jilani G, Haq MA (1999) Rice and wheat production in Pakistan with effective microorganisms. Am J Alternative Agric 14(01):30-36. doi:10.1017/S0889189300007980

Hussain T, Anjum AD, Tahir J (2002) Technology of beneficial microorganisms. Nature Farming and Environment 3:1-14

Iwaishi S (2001) Effect of organic fertilizer and effective microorganisms on growth, yield and quality of paddy-rice varieties. J Crop Prod 3(1):269-273. doi:10.1300/J144v03n01 22

Javaid A (2006) Foliar application of effective microorganisms on pea as an alternative fertilizer. Agron Sustain Dev 26(4):257-262

Javaid A (2010) Beneficial microorganisms for sustainable agriculture. In: Lichtfouse E (ed) Genetic engineering, biofertilisation, soil quality and organic farming. Sustainable agriculture reviews, vol 4. Springer, Netherlands, pp 347-369. doi:10.1007/978-90-4818741-6 12

Javaid A (2011) Effects of biofertilizers combined with different soil amendments on potted rice plants. Chil J Ag Res 71(1):157-163

Javaid A, Bajwa R (2011a) Effect of effective microorganism application on crop growth, yield, and nutrition in Vigna radiata (L.) Wilczek in different soil amendment systems. Commun Soil Sci Plant Anal 42(17):2112-2121. doi:10.1080/00103624.2011.596240

Javaid A, Bajwa R (2011b) Field evaluation of effective microorganisms (EM) application for growth, nodulation, and nutrition of mung bean. Turk J Agric For 35(4):443-452. doi:10.3906/tar-1001-599

Javaid A, Shah MBM (2010) Growth and yield response of wheat to EM (effective microorganisms) and parthenium green manure. Afr $\mathrm{J}$ Biotechnol 9(23):3373-3381
Lin DL (1991) Nature farming in Taiwan: effect of EM on growth and yield of paddy rice. In: Par JF, Hornick SB, Simpson ME (eds). The 2nd conference on Effective Microorganisms ${ }^{\mathrm{TM}}$ vol 2. International Conference on Kyusei Nature Farming, Brazil, p 196

Mayer J, Scheid S, Widmer F, Fließbach A, Oberholzer H-R (2010) How effective are 'Effective microorganisms ${ }^{\circledR}$ (EM)'? Results from a field study in temperate climate. Appl Soil Ecol 46(2):230-239. doi:10.1016/j.apsoil.2010.08.007

Okorski A, Olszewski J, Głowacka K, Okorska S, Pszczółkowska A (2010) The effect of the application of the biological control agent EM1 on gas exchange parameters and productivity of Pisum sativum L. infected with Fusarium oxysporum Schlecht. Acta Agrobot 63(2):105-115

Pineda A, Zheng S-J, van Loon JJA, Pieterse CMJ, Dicke M (2010) Helping plants to deal with insects: the role of beneficial soil-borne microbes. Trends Plant Sci 15(9):507-514. doi:10.1016/j.tplants. 2010.05.007

Pozo MJ, Azcon-Aguilar C (2007) Unraveling mycorrhiza-induced resistance. Curr Opin Plant Biol 10(4):393-398

Schenck zu Schweinsberg-Mickan M, Müller T (2009) Impact of effective microorganisms and other biofertilizers on soil microbial characteristics, organic-matter decomposition, and plant growth. J Plant Nutr Soil Sci 172(5):704-712. doi:10.1002/jpln.200800021

Thaler JS, Humphrey PT, Whiteman NK (2012) Evolution of jasmonate and salicylate signal crosstalk. Trends Plant Sci 17(5):260-270

Ton J, De Vos M, Robben C, Buchala A, Metraux JP, Van Loon LC, Pieterse CMJ (2002) Characterization of Arabidopsis enhanced disease susceptibility mutants that are affected in systemically induced resistance. Plant J 29(1):11-21

Ursi Ventura M, Ototumi A, Neves PMOJ (2006) Feeding preference of Diabrotica speciosa (Ger.) (Coleoptera: Chrysomelidae) by broccoli leaves from natural, organic and conventional farming systems. Semina Ciências Agrárias 27(1):125-132

Van der Heijden MGA, Bardgett RD, Van Straalen NM (2008) The unseen majority: soil microbes as drivers of plant diversity and productivity in terrestrial ecosystems. Ecol Lett 11(3):296-310. doi:10.1111/j.1461-0248.2007.01139.x

van Vliet PCJ, Bloem J, de Goede RGM (2006) Microbial diversity, nitrogen loss and grass production after addition of effective micro-organisms ${ }^{\circledR}$ (EM) to slurry manure. Appl Soil Ecol 32(2): 188-198. doi:10.1016/j.apsoil.2005.07.001

Wardle DA, Yeates GW, Williamson WM, Bonner KI, Barker GM (2004) Linking aboveground and belowground communities: the indirect influence of aphid species identity and diversity on a three trophic level soil food web. Oikos 107(2):283-294

$\mathrm{Xu} \mathrm{H}-\mathrm{L}$ (2001) Effects of a microbial inoculant and organic fertilizers on the growth, photosynthesis and yield of sweet corn. J Crop Prod 3(1):183-214. doi: $10.1300 / J 144 \mathrm{v} 03 \mathrm{n} 0116$

Xu H-L, Wang R, Mriddha MAU (2000) Effects of organic fertilizers and a microbial inoculant on leaf photosynthesis and fruit yield and quality of tomato plants. J Crop Prod 3(1):173-182 